\title{
Erratum to: A Probabilistic Proof of the Fundamental Gap Conjecture Via the Coupling by Reflection
}

\author{
Fuzhou Gong ${ }^{1} \cdot$ Huaiqian $\mathrm{Li}^{2} \cdot$ Dejun Luo'
}

Published online: 14 January 2016

(C) Springer Science+Business Media Dordrecht 2016

\section{Erratum to: Potential Anal}

\section{DOI 10.1007/s11118-015-9476-3}

In [3, Proposition A.7], we constructed a smooth function $u_{0}(x)=e^{-c_{2}|x|^{2} / 2} \rho^{\kappa}(x)$ on $\bar{\Omega}$ which admits $\log \tilde{\phi}_{0}$ as its modulus of log-concavity, that is, for all $x, y \in \Omega, x \neq y$,

$$
\left\langle\nabla \log u_{0}(y)-\nabla \log u_{0}(x), \frac{y-x}{|y-x|}\right\rangle \leq 2\left(\log \tilde{\phi}_{0}\right)^{\prime}\left(\frac{|x-y|}{2}\right) .
$$

However, from the choice of $\kappa$ in the proof of [3, Lemma A.6], we see that the function $u_{0}$ has zero gradient on the boundary $\partial \Omega$. Therefore, it does not satisfy the assumptions of [3, Lemma A.2]. In the following, we shall prove that, for any $0<\kappa<1$, there exists a function $u_{\kappa} \in C^{\infty}\left(\bar{\Omega}, \mathbb{R}_{+}\right)$with non-zero gradient on $\partial \Omega$, which admits $\kappa \log \tilde{\phi}_{0}$ as its modulus of log-concavity. This is sufficient to establish the log-concavity estimate for the ground state, see Theorem 5 below. We use the same notations as in [3]. In particular, $\rho_{\partial \Omega}: \bar{\Omega} \rightarrow \mathbb{R}_{+}$is the distance function to the boundary $\partial \Omega$ and $\rho \in C^{\infty}(\bar{\Omega})$ coincides with $\rho_{\partial \Omega}$ on $\partial_{r_{0}} \Omega=\left\{x \in \bar{\Omega}: \rho_{\partial \Omega}(x) \leq r_{0}\right\}$. Here we may assume that $r_{0}>0$ is small enough

The online version of the original article can be found at http://dx.doi.org/10.1007/s11118-015-9476-3.

Dejun Luo

luodj@amss.ac.cn

Fuzhou Gong

fzgong@amt.ac.cn

Huaiqian Li

hqlee@scu.edu.cn

1 Institute of Applied Mathematics, Academy of Mathematics and Systems Science,

Chinese Academy of Sciences, Beijing 100190, China

2 School of Mathematics, Sichuan University, Chengdu 610064, China 
such that $\rho_{\partial \Omega}$ is smooth on $\partial_{2 r_{0}} \Omega$. $\rho$ can be chosen as $f \circ \rho_{\partial \Omega}$, where $f: \mathbb{R}_{+} \rightarrow \mathbb{R}_{+}$is a smooth increasing function such that $f(t)=t$ for $t \in\left[0, r_{0}\right]$ and $f(t)=3 r_{0} / 2$ for $t \geq 2 r_{0}$.

First, the assertion of [3, Lemma A.5] can be strengthened as follows.

Lemma 1 For any $\theta_{0} \in(0,1)$, we can find $\varepsilon_{0} \in\left(0, r_{0}\right]$ such that

$$
\left\langle\nabla \rho(x), \frac{y-x}{|y-x|}\right\rangle \geq \theta_{0}, \quad \text { for all } x, y \in \Omega \text { with }|x-y| \geq D-\varepsilon_{0} \text {. }
$$

Proof We consider the function

$$
F(x, y)=\left\langle\nabla \rho(x), \frac{y-x}{|y-x|}\right\rangle, \quad x, y \in \bar{\Omega} \text { and } x \neq y,
$$

which is continuous on the closed set $\{(x, y) \in \bar{\Omega} \times \bar{\Omega}:|x-y| \geq D / 2\}$. Let $\bar{S}$ be the closed subset of $\bar{\Omega} \times \bar{\Omega}$ consisting of pairs of points such that their distance is exactly $D$, i.e. $\bar{S}=\{(x, y) \in \bar{\Omega} \times \bar{\Omega}:|x-y|=D\}$. If $\left(x_{0}, y_{0}\right) \in \bar{S}$, then we have $x_{0}, y_{0} \in \partial \Omega$ and $\nabla \rho\left(x_{0}\right)=\nabla \rho_{\partial \Omega}\left(x_{0}\right)=N\left(x_{0}\right)=\frac{y_{0}-x_{0}}{\left|y_{0}-x_{0}\right|}$, thus $F\left(x_{0}, y_{0}\right)=1$.

For sufficiently small $\varepsilon>0$, we define

$$
F_{\varepsilon}=\min \{F(x, y): x, y \in \bar{\Omega} \text { with }|x-y| \geq D-\varepsilon\} .
$$

Then $F_{\varepsilon}$ increases as $\varepsilon$ tends to 0 . Note that if $x \in \partial \Omega$, then $|\nabla \rho(x)|=|N(x)|=1$, which implies $F_{\varepsilon} \leq 1$. Thus the limit $\bar{\theta}:=\lim _{\varepsilon \rightarrow 0} F_{\varepsilon}$ exists and $\bar{\theta} \leq 1$. If $\bar{\theta}<1$, then we can find a sequence $\left\{\left(x_{n}, y_{n}\right)\right\}_{n \geq 1} \subset \bar{\Omega} \times \bar{\Omega}$ such that $\left|x_{n}-y_{n}\right| \rightarrow D$ and $F\left(x_{n}, y_{n}\right) \leq \bar{\theta}$. Extracting a subsequence if necessary, we may assume $\left(x_{n}, y_{n}\right) \rightarrow(\bar{x}, \bar{y})$ as $n \rightarrow \infty$, hence $(\bar{x}, \bar{y}) \in \bar{S}$ and $F(\bar{x}, \bar{y}) \leq \bar{\theta}<1$. This is a contradiction. Therefore, $\lim _{\varepsilon \rightarrow 0} F_{\varepsilon}=1$ which yields the desired result.

The next result is used to replace [3, Lemma A.6].

Lemma 2 For any $\kappa \in(0,1)$, there exists a positive constant $c_{2}$, depending on $\kappa$, such that for all $x, y \in \Omega, x \neq y$,

$$
\left\langle\nabla \log \rho(y)-\nabla \log \rho(x), \frac{y-x}{|y-x|}\right\rangle \leq 2 \kappa\left(\log \tilde{\phi}_{0}\right)^{\prime}\left(\frac{|x-y|}{2}\right)+c_{2}|x-y| .
$$

Proof We fix an arbitrary $\kappa \in(0,1)$. Take $\theta_{0} \in(\kappa, 1)$ and $\alpha>0$ such that $\kappa=4 \theta_{0} /(4+\alpha)$. Let $\varepsilon_{0}>0$ be determined as in Lemma 1 for this $\theta_{0}$. Set $\varepsilon_{1}=\min \left\{\varepsilon_{0}, \alpha / 2 c_{1}\right\}$, where $c_{1}$ is given in [3, Proposition A.4].

Fix any $x, y \in \Omega, x \neq y$. There are two different cases. First, if $|y-x|>D-\varepsilon_{1}$, then we can apply Lemma 1 to get

$$
\begin{aligned}
\left\langle\nabla \log \rho(y)-\nabla \log \rho(x), \frac{y-x}{|y-x|}\right\rangle & =-\left[\left\langle\frac{\nabla \rho(y)}{\rho(y)}, \frac{x-y}{|x-y|}\right\rangle+\left\langle\frac{\nabla \rho(x)}{\rho(x)}, \frac{y-x}{|y-x|}\right\rangle\right] \\
& \leq-\theta_{0}\left[\frac{1}{\rho(y)}+\frac{1}{\rho(x)}\right] .
\end{aligned}
$$

Suppose the straight line passing from $x$ to $y$ intersects the boundary $\partial \Omega$ first at the point $\hat{x}$ and then at $\hat{y}$. We have

$$
\rho_{\partial \Omega}(x)+|x-y|+\rho_{\partial \Omega}(y) \leq|\hat{x}-x|+|x-y|+|y-\hat{y}|=|\hat{x}-\hat{y}| \leq D .
$$


Hence $\rho_{\partial \Omega}(x)+\rho_{\partial \Omega}(y) \leq D-|x-y|<\varepsilon_{1}$, thus $x, y \in \partial_{\varepsilon_{1}} \Omega \subset \partial_{r_{0}} \Omega$, which implies $\rho(x)=\rho_{\partial \Omega}(x)$ and $\rho(y)=\rho_{\partial \Omega}(y)$. Therefore,

$$
\rho(x)+\rho(y) \leq D-|x-y|<\varepsilon_{1} .
$$

As a result,

$$
(\rho(x)+\rho(y))(D-|x-y|) \geq(\rho(x)+\rho(y))^{2} \geq 4 \rho(x) \rho(y),
$$

which, together with [3, Proposition A.4], implies

$$
\begin{aligned}
-\left[\frac{1}{\rho(y)}+\frac{1}{\rho(x)}\right] & \leq-\frac{4}{D-|x-y|}=-\frac{4}{4+\alpha}\left(\frac{4}{D-|x-y|}+\frac{\alpha}{D-|x-y|}\right) \\
& \leq \frac{4}{4+\alpha}\left[2\left(\log \tilde{\phi}_{0}\right)^{\prime}\left(\frac{|x-y|}{2}\right)+2 c_{1}-\frac{\alpha}{D-|x-y|}\right] \\
& \leq \frac{8}{4+\alpha}\left(\log \tilde{\phi}_{0}\right)^{\prime}\left(\frac{|x-y|}{2}\right),
\end{aligned}
$$

where the last inequality follows from Eq. 3 and $\varepsilon_{1} \leq \alpha / 2 c_{1}$. Combining this inequality with Eq. 2, we arrive at

$$
\left\langle\nabla \log \rho(y)-\nabla \log \rho(x), \frac{y-x}{|y-x|}\right\rangle \leq 2 \kappa\left(\log \tilde{\phi}_{0}\right)^{\prime}\left(\frac{|x-y|}{2}\right) .
$$

Therefore, we obtain (1) with $c_{2}=0$ in this case.

Next, we consider the second case that $0<|x-y| \leq D-\varepsilon_{1}$. Noticing that

$$
\left(\log \tilde{\phi}_{0}\right)^{\prime \prime}(t)=\frac{\tilde{\phi}_{0} \tilde{\phi}_{0}^{\prime \prime}-\left(\tilde{\phi}_{0}^{\prime}\right)^{2}}{\tilde{\phi}_{0}^{2}}(t)=\tilde{V}(t)-\tilde{\lambda}_{0}-\left(\frac{\tilde{\phi}_{0}^{\prime}}{\tilde{\phi}_{0}}\right)^{2}(t),
$$

we can find $\bar{c}>0$ such that

$$
\left|\left(\log \tilde{\phi}_{0}\right)^{\prime \prime}(t)\right| \leq \bar{c}, \quad \text { for all } t \in\left[0,\left(D-\varepsilon_{1}\right) / 2\right] .
$$

Moreover, $\tilde{\phi}_{0}^{\prime}(0)=0$ since $\tilde{\phi}_{0}$ is an even function. Thus, for any $t \in\left[0,\left(D-\varepsilon_{1}\right) / 2\right]$,

$$
\left|\left(\log \tilde{\phi}_{0}\right)^{\prime}(t)\right|=\left|\left(\log \tilde{\phi}_{0}\right)^{\prime}(t)-\left(\log \tilde{\phi}_{0}\right)^{\prime}(0)\right| \leq \bar{c} t .
$$

Finally, it is clear (cf. [3, Lemma A.2(4)]) that $\nabla^{2} \log \rho \leq \hat{c}$ for some $\hat{c} \geq 0$; hence

$$
\begin{aligned}
\left\langle\nabla \log \rho(y)-\nabla \log \rho(x), \frac{y-x}{|y-x|}\right\rangle & =\frac{1}{|x-y|} \int_{0}^{1}\left\langle\left.\nabla^{2} \log \rho\right|_{x+t(y-x)}(y-x), y-x\right\rangle \mathrm{d} t \\
& \leq \hat{c}|x-y| .
\end{aligned}
$$

Combining the above inequality with Eq. 4, we get (1) with $c_{2}=\bar{c}+\hat{c}$ in the second case.

We can now prove

Proposition 3 Fix any $\kappa \in(0,1)$ and let $c_{2}=c_{2}(\kappa)$ be given in Lemma 2. Define the function

$$
u_{\kappa}(x)=e^{-c_{2}|x|^{2} / 2} \rho(x), \quad x \in \bar{\Omega} .
$$

Then for all $x, y \in \Omega, x \neq y$, we have

$$
\left\langle\nabla \log u_{\kappa}(y)-\nabla \log u_{\kappa}(x), \frac{y-x}{|y-x|}\right\rangle \leq 2 \kappa\left(\log \tilde{\phi}_{0}\right)^{\prime}\left(\frac{|x-y|}{2}\right) .
$$


Proof By the definition of $u_{\kappa}$, we have

$$
\nabla \log u_{\kappa}(x)=-c_{2} x+\nabla \log \rho(x) .
$$

Thus, it follows from Lemma 2 that

$$
\begin{aligned}
\left\langle\nabla \log u_{\kappa}(y)-\nabla \log u_{\kappa}(x), \frac{y-x}{|y-x|}\right\rangle & =-c_{2}|y-x|+\left\langle\nabla \log \rho(y)-\nabla \log \rho(x), \frac{y-x}{|y-x|}\right\rangle \\
& \leq 2 \kappa\left(\log \tilde{\phi}_{0}\right)^{\prime}\left(\frac{|x-y|}{2}\right) .
\end{aligned}
$$

The proof is complete.

Next, analogous results of [3, Proposition A.8] still hold after small modifications.

Proposition 4 For any $\kappa$ in a small left neighborhood of 1 , there exists a smooth function $\psi_{\kappa} \in C^{\infty}([0, D / 2])$ satisfying $\psi_{\kappa}(0)=0$ and

(i) $\psi_{\kappa}^{\prime \prime}+2 \psi_{\kappa} \psi_{\kappa}^{\prime}-\tilde{V}^{\prime}=0$ on $[0, D / 2)$;

(ii) as $\kappa \uparrow 1, \psi_{\kappa}$ converges to $\left(\log \tilde{\phi}_{0}\right)^{\prime}$ pointwise on $[0, D / 2)$;

(iii) $\quad \psi_{\kappa}$ is a modulus of log-concavity of $u_{\kappa}$ constructed in Proposition 3.

Proof We may assume $\tilde{V}$ is nonnegative on $[-D / 2, D / 2]$, since adding a positive constant $C$ to $\tilde{V}$ does not change the eigen-functions $\left\{\tilde{\phi}_{i}\right\}_{i>0}$, with the corresponding eigenvalues $\left\{\tilde{\lambda}_{i}+C\right\}_{i \geq 0}$. We follow the idea in the proof of [1, Proposition 3.2]. Note that $\psi:=\left(\log \tilde{\phi}_{0}\right)^{\prime}$ satisfies $\psi(0)=0$ and $\psi^{\prime}=\tilde{V}-\tilde{\lambda}_{0}-\psi^{2}$ on the interval $(-D / 2, D / 2)$. Letting $q=$ $\arctan \psi$, then $q(0)=0$ and

$$
q^{\prime}-\left(\tilde{V}-\tilde{\lambda}_{0}\right) \cos ^{2} q+\sin ^{2} q=0 \quad \text { on }[-D / 2, D / 2] .
$$

Here the derivatives of $q$ at the endpoints are understood as right or left derivative, respectively. For $\kappa \in(0,1)$, we consider the first order ODE

$$
\begin{aligned}
\frac{\partial q}{\partial z}-\left[(\tilde{V} / \kappa)-\tilde{\lambda}_{0}\right] \cos ^{2} q+\kappa \sin ^{2} q & =0, \quad|z| \leq D / 2 ; \\
q(0, \kappa) & =0 .
\end{aligned}
$$

By the ODE comparison theorem (cf. [2, p.23, Corollary 1]), $q$ is strictly decreasing in $\kappa$ for $z>0$. The choice $\kappa=1$ corresponds to $q=\arctan \psi$; hence $q(D / 2,1)=-\pi / 2$ and $q(z, 1) \in(-\pi / 2, \pi / 2)$ for $0 \leq z<D / 2$.

Because $q(D / 2, \kappa)$ is strictly decreasing in $\kappa$ and $q(D / 2,1)=-\pi / 2$, for $\kappa<1$ in a small neighborhood of 1 , we have $q(D / 2, \kappa)>-\pi / 2$ and $q(z, \kappa) \in(-\pi / 2, \pi / 2)$ for $0 \leq z<D / 2$. Set $\psi_{\kappa}(z)=\kappa \tan q(z, \kappa),|z| \leq D / 2$. Direct calculations show that $\psi_{\kappa}$ satisfies (i). Since $\kappa<1$,

$$
\tan q(z, \kappa)>\tan q(z, 1)=\psi(z)=\left(\log \tilde{\phi}_{0}\right)^{\prime}(z) \quad \text { for } z \in(0, D / 2) .
$$

Thus by Proposition $3, \psi_{\kappa}$ is a modulus of concavity of $\log u_{\kappa}$ for all $\kappa$ in a small left neighborhood of 1 , which means (iii) holds. It remains to check the second assertion. By the continuous dependence on $\kappa$ of the solution $q(z, \kappa)$ (cf. [2, p.107, Corollary]), for any $z \in(0, D / 2), \tan q(z, \kappa) \rightarrow \tan q(z, 1)$ as $\kappa$ grows to 1 , which implies the pointwise convergence of $\psi_{\kappa}$ to $\left(\log \tilde{\phi}_{0}\right)^{\prime}$ on $[0, D / 2)$. Therefore, assertion (ii) is also verified.

Finally, we show that the result of [3, Theorem 2.6] is still true. 
Theorem 5 (Sharp log-concavity estimate). Assume that the potential $V$ has a modulus of convexity $\tilde{V}$. Then $\log \tilde{\phi}_{0}$ is a modulus of concavity for $\log \phi_{0}$.

Proof Fix any $\kappa \in(0,1)$. Let $u_{\kappa} \in C^{\infty}(\bar{\Omega})$ and $\psi_{\kappa} \in C^{\infty}([0, D / 2])$ be the functions constructed in Propositions 3 and 4, respectively. Applying [3, Theorem 2.4] with $u_{0}=u_{\kappa}$ and $\psi=\psi_{\kappa}$, we obtain that for all $x, y \in \Omega, x \neq y$,

$$
\left\langle\nabla \log u(t, x)-\nabla \log u(t, y), \frac{x-y}{|x-y|}\right\rangle \leq 2 \psi_{\kappa}\left(\frac{|x-y|}{2}\right)
$$

for any $t>0$. We deduce from the approximation lemma (see [3, Lemma A.3]) that for all $x \in \Omega$,

$\lim _{t \rightarrow \infty} \nabla \log u(t, x)=\lim _{t \rightarrow \infty} \frac{\nabla u(t, x)}{u(t, x)}=\lim _{t \rightarrow \infty} \frac{\nabla\left(e^{\lambda_{0} t} u(t, x)\right)}{e^{\lambda_{0} t} u(t, x)}=\frac{\nabla\left(a_{0} \phi_{0}(x)\right)}{a_{0} \phi_{0}(x)}=\nabla \log \phi_{0}(x)$.

Therefore, letting $t \rightarrow \infty$ in Eq. 6 yields

$$
\left\langle\nabla \log \phi_{0}(x)-\nabla \log \phi_{0}(y), \frac{x-y}{|x-y|}\right\rangle \leq 2 \psi_{\kappa}\left(\frac{|x-y|}{2}\right) .
$$

By Proposition 4(ii), letting $\kappa \uparrow 1$ in the above inequality yields that for all $x, y \in \Omega, x \neq y$,

$$
\left\langle\nabla \log \phi_{0}(x)-\nabla \log \phi_{0}(y), \frac{x-y}{|x-y|}\right\rangle \leq 2\left(\log \tilde{\phi}_{0}\right)^{\prime}\left(\frac{|x-y|}{2}\right) .
$$

The proof is complete.

Acknowledgments We are very grateful to Abraham Ng from the University of Sydney for pointing us that the function $u_{0}$ constructed in [3, Proposition A.7] has zero-gradient on the boundary $\partial \Omega$.

\section{References}

1. Andrews, B., Clutterbuck, J.: Proof of the fundamental gap conjecture. J. Amer. Math. Soc. 24(3), 899916 (2011)

2. Birkhoff, G., Rota, G.-C.: Ordinary Differential Equations. 2nd edn. Blaisdell Publishing Co. Ginn and Co., Waltham, Mass.-Toronto, Ont.-London (1969)

3. Gong, F.-Z., Li, H.-Q., Luo, D.-J.: A probabilistic proof of the fundamental gap conjecture via the coupling by reflection. Potential Anal. doi:10.1007/s11118-015-9476-3 